\title{
How does adopting a Growth Mindset Improve Academic Performance? Probing the Underlying Mechanisms in a Nationally- Representative Sample
}

\author{
Maithreyi Gopalan ${ }^{1}$ and David Yeager ${ }^{2}$
}

September 13, 2019

\begin{abstract}
Students who are taught that intellectual abilities are not fixed but can be developed-a growth mindset of intelligence-show improvements in their academic outcomes. Specifically, the mindset treatment effects are strongest for low-achieving students and vary across school contexts, as demonstrated by several lab and field-experimental studies including the largest randomized control trial (RCT) evaluation in a nationallyrepresentative sample of $9^{\text {th }}$ graders in the US as shown by the National Study of Learning Mindsets (NSLM). Yet, the mechanisms through which a growth mindset brings about positive academic outcomes remains unclear. Past research posits a role for challenge-seeking/learning-oriented behaviors exhibited by students exposed to a growth mindset intervention. However, research illuminating this pathway from mindset to academic outcomes through student behaviors are sparse. For the first time, given the multisite RCT design and the inclusion of a rich set of mediators-especially, a mediator measured using a novel behavioral task that elicits learning-oriented behavior in the NSLM, rather than relying on just self-reported mindset measures, this research tests the complete mediational pathway and highlights a key mechanism through which growth mindset interventions may work.
\end{abstract}

Keywords: growth mindset, instrumental variable, psychological interventions, randomized control trials, motivation, causal mechanisms

\footnotetext{
${ }^{1}$ The Pennsylvania State University; Corresponding Author Email: smg632@psu.edu; ${ }^{2}$ David Yeager; University of Texas, Austin; Email:dyeager@utexas.edu. This manuscript uses data from the National Study of Learning Mindsets (PI: D. Yeager; Co-Is: R. Crosnoe, C. Dweck, C. Muller, B. Schneider, \& G. Walton), which was made possible through methods and data systems created by the Project for Education Research That Scales (PERTS), data collection carried out by ICF International and led by Kate Flint, Alice Roberts, Jill Trott, and Ronaldo lachan, meetings hosted by the Mindset Scholars Network at the Center for Advanced Study in the Behavioral Sciences, assistance from C. Hulleman, R. Ferguson, M. Shankar, T. Brock, C. Romero, D. Paunesku, C. Macrander, T. Wilson, E. Konar, M. Weiss, E. Tipton, and A. Duckworth, and funding from the Raikes Foundation, the William T. Grant Foundation, the Spencer Foundation, the Bezos Family Foundation, the Character Lab, the Houston Endowment, Angela Duckworth (personal gift), and the President and Dean of Humanities and Social Sciences at Stanford University. These data were made available to the researchers through the Population Research Center at The University of Texas at Austin which is supported by grant, P2CHD042849, awarded by the Eunice Kennedy Shriver National Institute of Health and Child Development. Opinions reflect those of the authors and do not necessarily reflect those of the granting agencies.
} 


\section{Introduction}

When adolescents are taught that intellectual abilities are not fixed but can be developed—a growth mindset of intelligence-they show improvements in their academic outcomes as evidenced by several lab and field-experimental studies (see Dweck \& Yeager, 2019). However, the key mechanisms of change, as evidenced by changes in behaviors, through which such favorable academic outcomes emerge, remain under-explored. For example, when students believe that they can improve their ability, do they work harder? Do they seek out challenging opportunities and other learning experiences? Do they persist when experiencing setbacks or challenging tasks that may be responsible for the positive change in their academic outcomes?

It is crucial to understand behavioral pathways, especially using rigorous, empirical causal inference techniques to further research in this area. Specifically, there has been a call to explore behavioral mediators (Miller, Dannals, \& Zlatev, 2017) that may lie in the pathway between the manipulated psychological states and final outcomes of interest (Walton \& Wilson, 2018). Indeed, such examination is integral to not just enhance the underlying psychological theory but also to generate policyrelevant insights that can provide practical implications; a task we have undertaken in this present study.

In this study, we integrate two research approaches-theoretically-driven, fieldexperimental manipulation of mindsets in psychology (Yeager \& Walton, 2011) and empirical advances in econometrics and program evaluation in estimating causal mediations using the potential outcomes framework (Neyman, Iwaszkiewicz, \& Kolodziejczyk, 1935; Rubin, 1974)—to advance mindset theory regarding the mediating 
processes that drive improvements in adolescents' motivation and academic achievement. Specifically, we examined the average causal effect of a key behavioral mediator—students' learning-oriented behavior—induced by the growth mindset intervention implemented in a nationally-representative sample across 65 schools in the US that links manipulated psychological states (the belief that intelligence is malleable) with academic outcomes (grade-point-average [GPA]).

\section{Theoretical and Empirical Background}

Decades of lab and field-experimental research have found that students' beliefs or implicit theories are associated with several social-cognitive, motivational, and selfregulatory processes that drive behavior change. In the context of mindset research, across several studies, researchers have illustrated a key role for causal attributions. For example, studies have shown that when students hold a fixed mindset-the belief that intellectual ability cannot change--they were more likely to make fixed trait attributions such as "I'm not smart at math" and adopt negative beliefs about effort (e.g., if "I have to try hard, it means I'm not smart") (see Leggett, 1986; Miele, Son, \& Metcalfe, 2013).

Such attributions and beliefs have been linked to goal-setting processes that have long shown to be key drivers of motivation (Burnette, O'Boyle, VanEpps, Pollack, \& Finkel, 2013). For example, in a meta-analytical review of mindset research, Burnette and colleague find that students who did not believe that their intellectual ability is malleable chose performance goals rather than learning goals (2013). Furthermore, studies have also shown that the attributions and effort beliefs that students make may become even more salient or consequential when they experience challenges or 
setbacks (Blackwell, Trzesniewski, \& Dweck, 2007). Specifically, past research shows that students with a fixed mindset exhibit defensive behaviors especially when experiencing a negative feedback. They chose not to take remedial actions that might help them improve or display "symbolic" responses to performance feedback that does not aim to repair their deficit. On the other hand, those students who adopt a growth mindset exhibited learning behaviors, which included remedial actions with the express goal of self-improvement (Nussbaum \& Dweck, 2008). In all, past research has suggested (but has not definitively shown using non-self-reported measures) that a growth mindset predicts more learning-oriented behaviors using rigorous methods.

In this vein, we hypothesized that students exposed to the growth mindset intervention would interpret struggles as learning opportunities rather than indications of fixed ability. Subsequently, they are likely to take on more challenges and may be more effortful and persistent. Because the behavioral task was explicitly designed to elicit students' behavior towards learning, the behavioral measure provides a unique insight into the pathway through which growth mindset interventions might take root to bring about the observed positive academic outcomes downstream. Therefore, there is a strong theoretical basis for our hypothesis on the key behavioral mediator thereby enabling us to illuminate the complete pathway from growth mindset leading to positive academic outcomes.

Econometrically, estimating causal mediating effects even in randomized control trials (RCTs), the "gold standard" of causal inference, is not straightforward because most mediators are not directly manipulated; rather they are measured. However, more recently, empirical methods have been developed to isolate the random variation in 
mediators induced by the randomized experimental manipulation (as well as the variation induced across experimental sites in a multisite RCT $)^{1}$ to identify the causal effects of various mediators on final outcomes of interest using a multi-site instrumental variables (IV) framework (Duncan, Morris, \& Rodrigues, 2011; S. Reardon \& Raudenbush, 2013). In effect, IV methods help isolate the "exogenous" variation in the key mediator of interest caused by random assignment to treatment or control groups (see Figure 1). Therefore, the randomization to different conditions, can be used as instruments to isolate the random variation in student behaviors. However, we also have to make several strict identification assumptions under this approach.

Alternatively, mediational analysis in psychology and other social sciences, has traditionally been based on a linear structural equation modeling framework (e.g. Baron \& Kenny, 1986; MacKinnon, 2008). Recently, this approach has been extended and placed within the counterfactual framework by including additional identifying assumptions (Imai, Keele, \& Tingley, 2010; Imai, Keele, \& Yamamoto, 2010). This has enabled causal interpretations of mediation effects under specific assumptions that are somewhat more relaxed than the IV assumptions. Additionally, these methods enable non-parametric estimation and a set of sensitivity analyses within the causal mediational frameworks. In alternative specifications, we include these analyses and test the robustness of our IV assumptions and resulting estimates.

Most recently, the NSLM estimated the average ITT effect as well as heterogeneity in the causal effects of adopting a growth mindset on academic outcomes

\footnotetext{
${ }^{1}$ A quick note on terminology. I use the term multisite randomized control trial to refer to a design where randomization occurred within each site across multiple sites.
} 
across school contexts (Yeager et al., 2019). However, no previous research has examined whether adoption of a growth mindset relates to learning-oriented behaviors-a theoretically-hypothesized mediator in the literature-especially, in a nationally-representative sample, or how the mechanisms may differ across contexts, nor has it exploited such variation to estimate the average causal effect of such learning-oriented behaviors induced by the growth mindset intervention on future academic outcomes.

\section{Data}

Data for the current study were drawn from the NSLM-a randomized trial that evaluated a growth mindset intervention—which teaches that intellectual abilities are not fixed but can be developed-in a nationally-representative sample of $9^{\text {th }}$ graders in US public schools (Yeager et al., forthcoming). In the beginning of the $9^{\text {th }}$ grade, students within 65 schools were assigned to either a growth mindset intervention or a control condition. Study participants were followed longitudinally post-intervention for a year with the main academic outcomes measured at the end of their $9^{\text {th }}$ grade and the beginning of $10^{\text {th }}$ grade. Our analysis sample was limited to students who had a valid grade point average calculated for the core academic subjects at the end of the $9^{\text {th }}$ grade.

\section{Measures}

Academic outcomes. We use the two pre-registered academic outcomes from the original NSLM: (1) Post-intervention GPA in core $9^{\text {th }}$ grade classes (Math, Science, 
English, and Social studies); and (2) Post-intervention GPA in Math and Science $9^{\text {th }}$ grade classes.

Learning-oriented behavior. We operationalize learning-oriented behavior-the key behavioral mediator-in our study using student-reports on a novel behavioral task "make-a-math-worksheet" task (see Yeager et al., 2019 for details). Students completed the task toward the end of the second session, after they completed the intervention (or control content). Students chose from math problems described as challenging (that offered the chance to learn a lot) or as easy (that would not lead to much learning). Students were informed that they could complete the problems at the end of the session if there was time. Past research provides strong support for the validity of this measure (Yeager et al., 2016).

Additional covariates. Additionally, we include student-level covariates—such as prior performance, demographics (student race, gender, parent education), and school fixed effects in alternative model specifications as appropriate.

\section{Methods}

Our primary goal was to estimate the average causal effect of students' learningoriented behavior on students' academic outcomes. Conceptually, the basic idea is to isolate a portion of variation in learning-oriented behaviors that is unrelated to unmeasured confound variables and then use only that portion to estimate the linkages between learning-oriented behaviors and achievement (see Figure 1 for the conceptual path model). To accomplish our analytic goals, we follow two primary steps. 
First, we isolate the exogenous portion of the learning-oriented behaviors which is induced by the randomized exposure to the growth mindset treatment condition using what is known as the first-stage regression. We specify the regression model as follows:

(1) $M_{i s}=\beta_{1} T_{i s}+\beta_{2} X_{i s}+\theta_{s}+\varepsilon_{i s}$

Where the dependent variable $M$ denotes the mediator, learning-oriented behavior, and $T$ denotes an indicator whether a student was assigned to the growth mindset condition ( $1=$ Yes, $0=$ No), vector $X$ denotes student-level demographics such as race, gender, baseline academic outcome and others, $\theta$ denotes school fixed effects, and $\varepsilon$ is a random error term. We estimate all models using ordinary least squares (OLS).

Second, the predicted values, $\widehat{M}_{l}$, are used in the second stage OLS regression as specified as follows after adjusting for standard errors (to account for using predicted values):

(2) $Y_{i}=\gamma_{1} \widehat{M}_{l}+\gamma_{2} X_{i s}+\phi_{s}+u_{i}$

Where the dependent variable $Y$ denotes the final outcome of interest, GPA for example, and $u$ is a random error term. Now the coefficient $\gamma$ provides an unbiased causal effect of the estimate of learning-oriented behaviors on academic achievement because it has been purged of all the endogeneity (i.e., the correlation between $u$ and M) by using the randomized treatment status as the instrumental variable.

Because the NSLM was a multisite, person-level randomized study, we can further expand the first and second stage regressions by incorporating site-level variation in the mediating mechanisms as well. We can therefore include site $\mathrm{x}$ treatment indicators as multiple instruments. We adapt equations (1) and (2) as follows: 
(3) $M_{i s}=\beta_{1}\left(T_{i s} \times \theta_{s}\right)+X_{i s}+\varepsilon_{i s}$

(4) $Y_{i}=\gamma_{1} \widehat{M}_{l}+\gamma_{2} X_{i s}+u_{i}$

\section{Preliminary Results}

Preliminary results show that, on average, the growth mindset intervention increased students' learning-oriented behaviors $(d=0.22, p<0.001)$. As expected, there was considerable heterogeneity in growth mindset induced learning-oriented behaviors across schools. We calculated this heterogeneity in the effect of treatment on the focal mediator following Bloom and colleagues' framework (2017). First, we specify a 2-level multilevel model (students clustered within schools) with school-specific intercepts and a random treatment coefficient that varies across schools. We estimate $\tau$, the standard deviation of treatment effect on the mediator across schools as 0.292 . We used restricted maximum likelihood method (REML) for estimating the random effect. To test whether $\tau$ is statistically different than zero, we use the Q-statistic proposed by Bloom et al. (2017). The Q-statistic, inspired from meta-analysis used to test cross-study impact variation, can be calculated as the sum of squared standard deviation of a precisionweighted treatment effect estimate on the mediator from site-specific models. Under the null, the Q-statistic follows a chi-square distribution with 64 degrees of freedom (there are 65 sites in the analytical sample). We estimate the Q-statistic to be 89.95 ( $p=$ $0.017)$.

Next, using the classic IV framework, we examine the first-stage results of the treatment on the mediator in the full sample using equation (1). As mentioned earlier, we find that a statistically significant treatment effect on the mediator $(\beta=0.957, F=$ 91.27, $p<0.001)$. In figure 3 , we also visually present the association between site- 
specific treatment effect on mediator and site-specific treatment effect on postintervention GPA across all sites in our analytic sample. A visual inspection shows what Reardon and Raudenbush (2013) call the compliance-effect covariance bias to be small. ${ }^{2}$ We find a very similar pattern when Empirical Bayes estimates of site-specific treatment effects on the mediator and outcomes are plotted. The average difference in treatment effects on post-intervention GPA that differ by one unit in the mediator (assuming no unobserved alternative mediators and that the treatment affects outcomes purely through the observed mediator) can be estimated asymptotically using these methods.

Given the positive first-stage results and strength of the instrument (F-stat >10), we use 2SLS methods to explore the average causal effect of learning-oriented behaviors on student outcomes approximately one year post-intervention. We find that, on average, the IV estimate is on students' core GPA is 0.063 points $(p=0.001)$ and STEM GPA is 0.07 points $(p<0.001)$, on average, across all schools. For low-achieving (defined based on prior achievement as defined in Yeager et al. (2019)) students, we find that the analogous IV estimate on students' core GPA is 0.124 points $(p=0.001)$ and STEM GPA is 0.126 points $(p=0.001)$, on average, across all schools.

Classic mediation analysis. As mentioned in the "Theoretical and Empirical Background" section, the IV framework we use in this paper contrasts with the nonparametric approach to encouragement designs developed in the methodological literature (Angrist, Imbens, \& Rubin, 1996) and applied to the causal mediation analysis

\footnotetext{
2 Under some assumptions, this bias can be numerically estimated in finite sample following (Reardon, Unlu, Zhu, \& Bloom, 2014); I hope to include this in the final draft of the paper.
} 
(Imai, Keele, \& Tingley, 2010; Imai, Keele, \& Yamamoto, 2010; Imai et al., 2013). We will include results from such a causal mediation analysis in the final paper as well.

\section{Discussion}

The present research adapted empirical methods stemming from advancements in econometrics and program evaluation to estimate the average causal effect of a key behavioral mediator of growth mindset interventions. Participants exposed to a growth mindset intervention - the belief that intelligence is malleable-exhibited higher learning-oriented behaviors compared with control participants. This effect, however, varies across the 65 schools in which the intervention was implemented. We used this variation to further understand the average causal effect of this behavioral mediator.

In other words, the present research helped illuminate how the growth mindset intervention affected academic performance many months later. The increased learning-oriented behavior induced by the intervention, in-turn, mediated the treatment effect on academic outcomes. Previous research did not consistently test for mediation of behavioral processes induced by growth mindset interventions on grades via nonself-reported measures, especially within a rigorous causal inference framework. In high school, adolescents may struggle with increased course loads, might receive negative feedback from teachers on assignments and course materials, and may face several other pressures to perform and get "college ready". Yet, our study shows that when they are encouraged to view intellectual ability as malleable, they may approach challenging tasks as another effort to learn and improve their skills and abilities rather than just displaying false competence. 
Finally, the present study aims to contribute to a new approach for measuring and estimating the causal effect of key mediators to understand the complete causal pathways of interventions better. First, thoughtful measurement of mediators must be operationalized using behavioral tasks that elicit hypothesized behavioral changes rather than just relying on self-report measures. Second, if those mediators can be measured across contexts within a multisite RCT, we can further exploit such contextvariation to understand the causal chain in improving adolescents' outcomes. 


\section{Tables and Figures}

Figure 1. Path Model

1

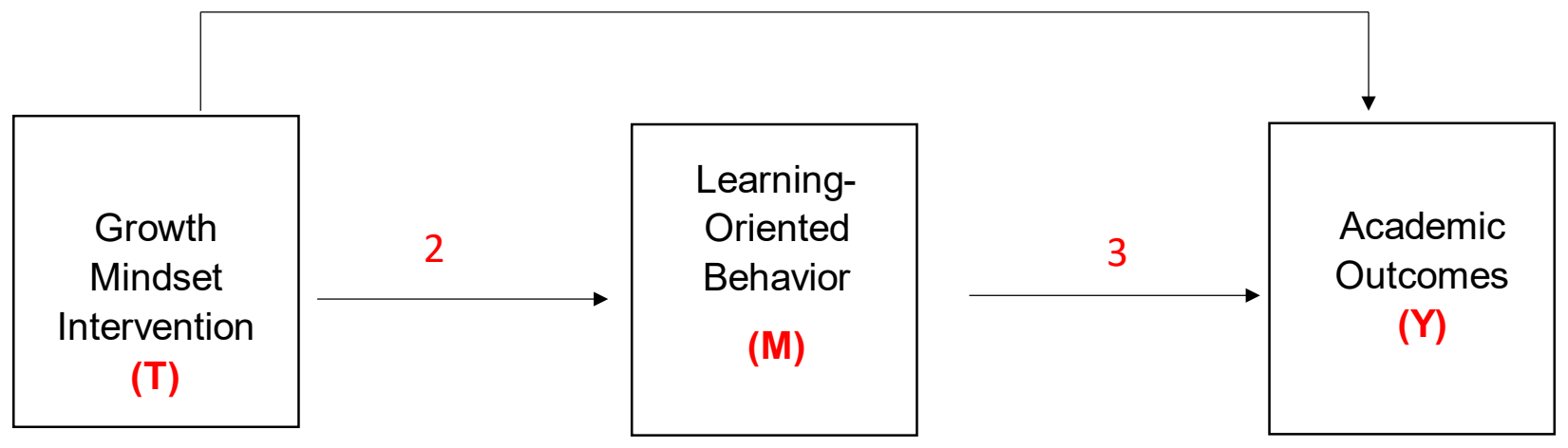


Figure 2. First-stage Results. Effects of Treatment on Learning-Oriented Behaviors

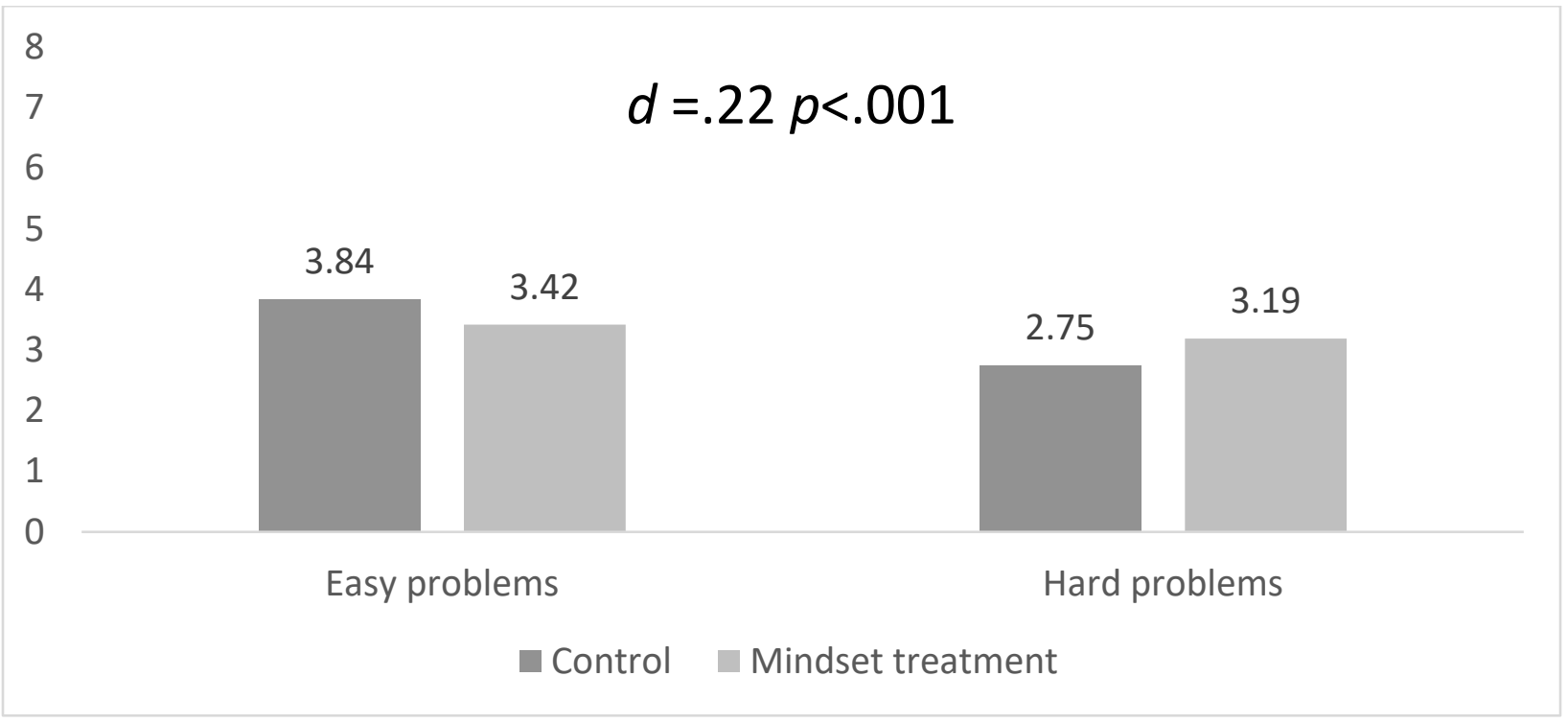


Figure 3. Association Between Site-Specific Effect of Treatment on Mediator and Site-Specific Effect of Treatment on Academic Outcomes

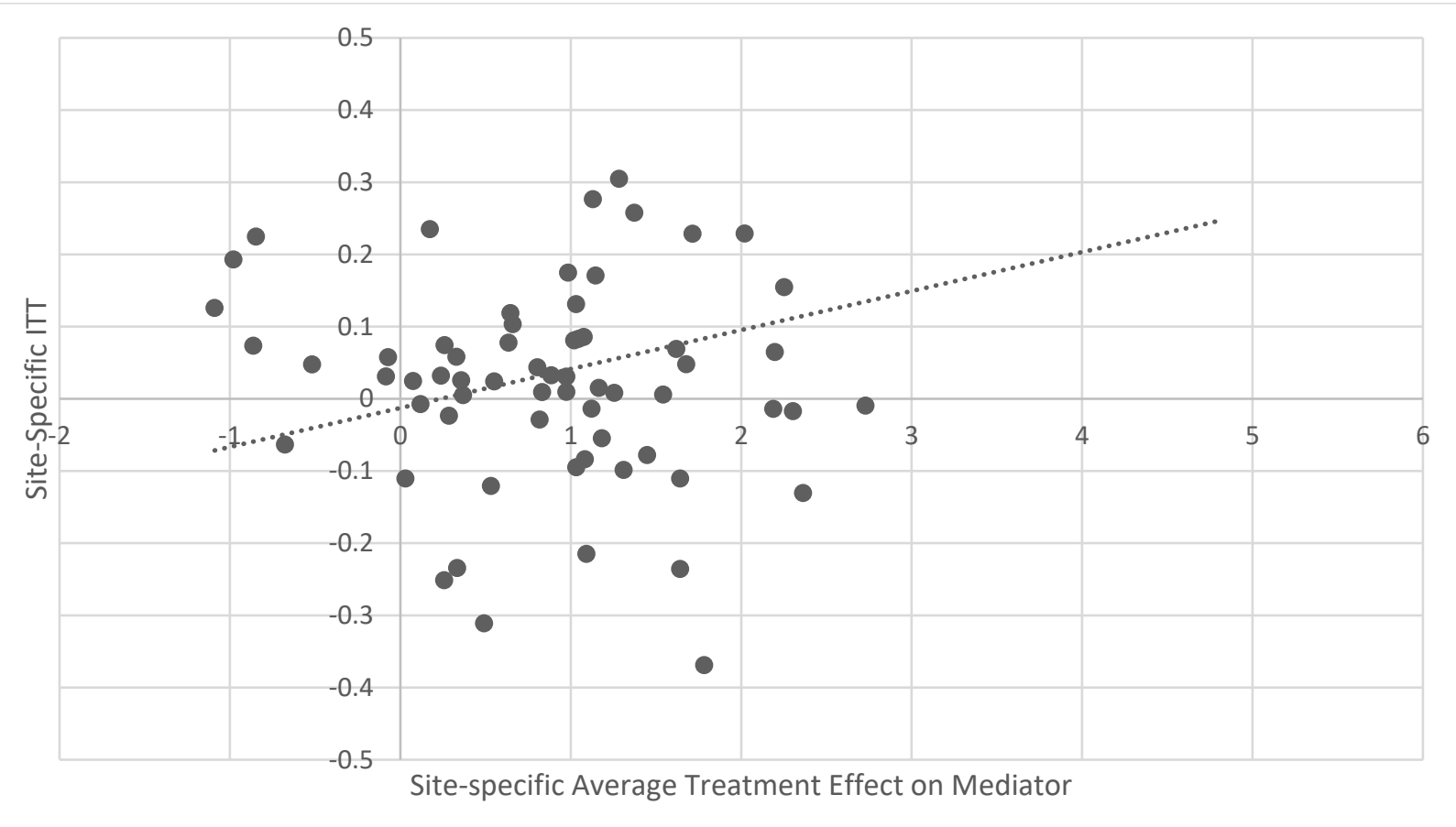


Table 1. Descriptive Statistics of Key Variables (Unweighted)

\begin{tabular}{lccc}
\hline \multirow{2}{*}{ Key Characteristics } & \multicolumn{3}{c}{ Analytic Sample } \\
\cline { 2 - 4 } & Mean & SD & $n$ \\
\hline Learning-Oriented Behavior & -0.66 & 3.78 & 11,628 \\
Academic Outcomes & & & \\
Post-Intervention Core GPA & & & \\
Post-Intervention Math/Science GPA & 2.60 & 1.07 & 12,488 \\
& 2.51 & 1.14 & 12,086 \\
Student Characteristics & & & \\
Female & & & \\
Maternal College Education & 0.49 & 0.50 & 13,421 \\
Asian & 0.29 & 0.45 & 13,421 \\
Black & 0.04 & 0.19 & 13,421 \\
Hispanic & 0.11 & 0.32 & 13,421 \\
White & 0.24 & 0.43 & 13,421 \\
& 0.43 & 0.50 & 13,421
\end{tabular}


Table 2. Preliminary Results (Full Sample)

Average Post-Intervention
GPA

\begin{tabular}{|c|c|c|c|c|}
\hline & OLS & $2 S L S$ & OLS & $2 S L S$ \\
\hline \multirow[t]{2}{*}{ Treatment } & $0.052^{* * *}$ & & $0.066^{* * *}$ & \\
\hline & $(0.016)$ & & $(0.018)$ & \\
\hline \multirow[t]{2}{*}{$\begin{array}{l}\text { Learning-Oriented } \\
\text { Behavior }\end{array}$} & & $0.063^{* * *}$ & & $0.070^{* * *}$ \\
\hline & & $(0.018)$ & & $(0.02)$ \\
\hline Site Fixed Effects & $X$ & $x$ & $x$ & $x$ \\
\hline Treatment as Instrument & & $\mathrm{X}$ & & $X$ \\
\hline $\begin{array}{l}\text { Post-stratification } \\
\text { Weights }\end{array}$ & $x$ & $x$ & $x$ & $x$ \\
\hline $\begin{array}{l}\text { Other Student-level } \\
\text { Covariates }\end{array}$ & $x$ & $x$ & $x$ & $x$ \\
\hline Number of Observations & 12,488 & 10,955 & 12,086 & 10,678 \\
\hline
\end{tabular}

Notes: Asterisks denote significance at the $0.1 \%\left({ }^{* * *}\right), 1 \%\left({ }^{* *}\right)$, and $5 \%\left({ }^{*}\right)$ levels. Robust standard errors in parentheses and clustered at the school-level. 
Table 3. Preliminary Results (At-risk Students)

Average Post-Intervention
GPA

\begin{tabular}{|c|c|c|c|c|}
\hline & OLS & $2 S L S$ & OLS & $2 S L S$ \\
\hline \multirow[t]{2}{*}{ Treatment } & $0.100^{* * *}$ & & $0.103^{* * *}$ & \\
\hline & $(0.024)$ & & $(0.027)$ & \\
\hline \multirow[t]{2}{*}{$\begin{array}{l}\text { Learning-Oriented } \\
\text { Behavior }\end{array}$} & & $0.123^{* * *}$ & & $0.127^{* * *}$ \\
\hline & & $(0.037)$ & & $(0.038)$ \\
\hline Site Fixed Effects & $x$ & $x$ & $x$ & $x$ \\
\hline Treatment as Instrument & & $x$ & & $x$ \\
\hline $\begin{array}{l}\text { Post-stratification } \\
\text { Weights }\end{array}$ & $x$ & $x$ & $x$ & $x$ \\
\hline $\begin{array}{l}\text { Other Student-level } \\
\text { Covariates }\end{array}$ & $x$ & $x$ & $x$ & $x$ \\
\hline Number of Observations & 6,325 & 5,434 & 6,146 & 5,313 \\
\hline
\end{tabular}

Notes: Asterisks denote significance at the $0.1 \%\left({ }^{* * *}\right), 1 \%\left({ }^{* *}\right)$, and $5 \%\left({ }^{*}\right)$ levels. Robust standard errors in parentheses and clustered at the school-level. 


\section{References}

Angrist, J. D., Imbens, G. W., \& Rubin, D. B. (1996). Identification of Causal Effects Using Instrumental Variables. Journal of the American Statistical Association, 91(434), 444-455. https://doi.org/10.2307/2291629

Baron, R. M., \& Kenny, D. A. (1986). The Moderator-Mediator Variable Distinction in Social Psychological Research: Conceptual, Strategic, and Statistical Considerations. Journal of Personality and Social Psychology, 51(6), 10.

Blackwell, L. S., Trzesniewski, K. H., \& Dweck, C. S. (2007). Implicit Theories of Intelligence Predict Achievement Across an Adolescent Transition: A Longitudinal Study and an Intervention. Child Development, 78(1), 246-263. https://doi.org/10.1111/j.1467-8624.2007.00995.x

Bloom, H. S., Raudenbush, S. W., Weiss, M. J., \& Porter, K. (2017). Using Multisite Experiments to Study Cross-Site Variation in Treatment Effects: A Hybrid Approach With Fixed Intercepts and a Random Treatment Coefficient. Journal of Research on Educational Effectiveness, 10(4), 817-842. https://doi.org/10.1080/19345747.2016.1264518

Burnette, J. L., O’Boyle, E. H., VanEpps, E. M., Pollack, J. M., \& Finkel, E. J. (2013). Mind-sets matter: A meta-analytic review of implicit theories and self-regulation. Psychological Bulletin, 139(3), 655-701. https://doi.org/10.1037/a0029531

Duncan, G. J., Morris, P. A., \& Rodrigues, C. (2011). Does money really matter? Estimating impacts of family income on young children's achievement with data from random-assignment experiments. Developmental Psychology, 47(5), 12631279. https://doi.org/10.1037/a0023875 
Dweck, C. S., \& Yeager, D. S. (2019). Mindsets: A View From Two Eras. Perspectives on Psychological Science, 14(3), 481-496.

https://doi.org/10.1177/1745691618804166

Imai, K., Keele, L., \& Tingley, D. (2010). A general approach to causal mediation analysis. Psychological Methods, 15(4), 309-334. https://doi.org/10.1037/a0020761

Imai, K., Keele, L., \& Yamamoto, T. (2010). Identification, Inference and Sensitivity Analysis for Causal Mediation Effects. Statistical Science, 25(1), 51-71.

Retrieved from https://www.jstor.org/stable/41058997

Imai, K., Tingley, D., \& Yamamoto, T. (2013). Experimental designs for identifying causal mechanisms: Experimental Designs for Identifying Causal Mechanisms. Journal of the Royal Statistical Society: Series A (Statistics in Society), 176(1), 5-51. https://doi.org/10.1111/j.1467-985X.2012.01032.x

Keele, L., Tingley, D., \& Yamamoto, T. (2015). IDENTIFYING MECHANISMS BEHIND POLICY INTERVENTIONS VIA CAUSAL MEDIATION ANALYSIS: Methods for Policy Analysis. Journal of Policy Analysis and Management, 34(4), 937-963. https://doi.org/10.1002/pam.21853

Leggett, E. L. (1986). Individual differences in effort/ability inference rules and goals: Implications for causal judgments. ProQuest Information \& Learning, US.

MacKinnon, D. P. (2008). Introduction to statistical mediation analysis. New York, NY: Taylor \& Francis Group/Lawrence Erlbaum Associates. 
Miele, D. B., Son, L. K., \& Metcalfe, J. (2013). Children's Naive Theories of Intelligence Influence Their Metacognitive Judgments. Child Development, 84(6), 1879-1886. https://doi.org/10.1111/cdev.12101

Miller, D. T., Dannals, J. E., \& Zlatev, J. J. (2017). Behavioral Processes in Long-Lag Intervention Studies. Perspectives on Psychological Science, 12(3), 454-467. https://doi.org/10.1177/1745691616681645

Neyman, J., Iwaszkiewicz, K., \& Kolodziejczyk, St. (1935). Statistical Problems in Agricultural Experimentation. Supplement to the Journal of the Royal Statistical Society, 2(2), 107-180. https://doi.org/10.2307/2983637

Nussbaum, A. D., \& Dweck, C. S. (2008). Defensiveness Versus Remediation: SelfTheories and Modes of Self-Esteem Maintenance. Personality and Social Psychology Bulletin, 34(5), 599-612. https://doi.org/10.1177/0146167207312960

Reardon, S. F., Unlu, F., Zhu, P., \& Bloom, H. S. (2014). Bias and Bias Correction in Multisite Instrumental Variables Analysis of Heterogeneous Mediator Effects. Journal of Educational and Behavioral Statistics, 39(1), 53-86. https://doi.org/10.3102/1076998613512525

Reardon, S., \& Raudenbush, S. (2013). Under What Assumptions Do Site-by-Treatment Instruments Identify Average Causal Effects? Sociological Methods \& Researc, 42(2), 143-163.

Rubin, D. B. (1974). Estimating causal effects of treatments in randomized and nonrandomized studies. Journal of Educational Psychology, 66(5), 688-701. https://doi.org/10.1037/h0037350 
Walton, G. M., \& Wilson, T. D. (2018). Wise interventions: Psychological remedies for social and personal problems. Psychological Review, 125(5), 617-655. https://doi.org/10.1037/rev0000115

Yeager, D. S., Hanselman, P., Walton, G. M., Murray, J. S., Crosnoe, R., Muller, C., ... Dweck, C. S. (2019). A national experiment reveals where a growth mindset improves achievement. Nature. https://doi.org/10.1038/s41586-019-1466-y

Yeager, D. S., Romero, C., Paunesku, D., Hulleman, C. S., Schneider, B., Hinojosa, C., ... Dweck, C. S. (2016). Using design thinking to improve psychological interventions: The case of the growth mindset during the transition to high school. Journal of Educational Psychology, 108(3), 374-391. https://doi.org/10.1037/edu0000098

Yeager, D. S., \& Walton, G. M. (2011). Social-psychological interventions in education: They're not magic. Review of Educational Research, 81(2), 267-301. 